\title{
WHY DOES REFLECTOR ENHANCE THE GAIN OF HELICAL ANTENNAS?
}

\author{
A.R. Djordjevic*, M.M. Ilic*, A.G. Zajic ${ }^{\dagger}$, D.I. Olcan*, and M.M. Nikolic* \\ *University of Belgrade, School of Electrical Engineering, P.O. Box 35-54, 11120 Belgrade, Serbia, \\ Email: edjordja@etf.bg.ac.yu \\ $\dagger$ Georgia Institute of Technology, 75 5th St. N.W., Atlanta, GA 30308, USA, Email: alenka@ece.gatech.edu
}

Keywords: Helical antennas, Antenna radiation patterns.

\begin{abstract}
We investigate uniformly-wound helical antennas above ground conductors of various shapes, with aim to determine factors that improve the antenna radiation pattern and gain. We show that the characteristics of helical antennas with large spillover fields can be improved using ground conductors (reflectors). This is achieved by intercepting unwanted radiation and redirecting it favorably.

\section{Introduction}

Helical antennas have been known for a long time [1], but the literature is overwhelmed with controversial information about their performance. In [2], we pointed out these discrepancies and presented the optimized design data for the helical antennas located above an infinite ground plane. In [3], we observed that the shape and dimensions of the ground conductor (reflector) have influence on helical antenna performance. In [4], we presented optimization results for helical antennas whose ground conductor has the shape of a truncated cone. Computed and measured results show that the cone substantially increases the gain of a helical antenna compared to an infinite ground plane. However, physical reasons behind this effect remained unknown. This paper investigates several effects that may explain why the reflector can enhance the gain of helical antennas.
\end{abstract}

First, we show that a large ground plane increases the bandwidth of some helical antennas. At lower frequencies, the ground reflects the field radiated from the helix downwards.

Second, we investigate fields radiated by various parts of the helix. We demonstrate that large sidelobes are produced by the current in the few lowest turns of the helix. Reducing the height of these turns improves the pattern because the radiation by the traveling-wave region of the helix becomes more pronounced.

Third, we point out that a properly designed ground conductor intercepts spillover (sidelobes) radiated by the helix and directs the energy in favorable directions.

Fourth, we conclude that a large truncated cone performs like a horn antenna. The helical antenna serves as the excitation of the horn. The main lobe of the radiation pattern of the horn is directed along the helix axis, so that the horn contributes to the radiation from the helix.

The paper is organized as follows. Section 2 reviews the known results about helical antennas above infinite ground plane and finite-size reflectors. Section 3 considers the current distribution along helical antennas to establish the feeding region and the traveling-wave region. Section 4 examines how an infinite ground plane influences the radiation pattern, by evaluating the fields produced by the currents and charges induced in the plane. Furthermore, the fields produced by the traveling-wave portion of the helix and the feeding region are examined. Finally, the contributions of cylindrical and conical reflectors are computed. Section 5 concludes the paper.

\section{Radiation pattern and gain of helical antennas with various reflectors}

In this section, we briefly review the known results about helical antennas above infinite ground plane and finite-size reflectors.

\subsection{Infinite ground plane}

Figure 1 shows a uniformly-wound helical antenna above infinite ground plane, which is assumed to radiate in the axial mode. The geometry of the helical antenna is defined by the number of turns $(N)$, helix pitch $(p)$, helix radius $(a)$, and wire radius $(r)$. The helix length is $L=N p$, the helix circumference is $C=2 \pi a$, and the pitch angle is $\alpha=\arctan (p / C)$.

According to the classical design data [5], the helical antenna operates in the axial mode in the frequency band where $3 / 4<C / \lambda<4 / 3$ ( $\lambda$ is the wavelength). The wire radius has practically no influence on the antenna characteristics in a wide range $0.0025<r / \lambda<0.025$. The optimal pitch angle is in within the relatively narrow range $12^{\circ}<\alpha<14^{\circ}$.

We choose an example that fits into the classical design data. A helical antenna is designed to operate near $1.7 \mathrm{GHz}$. The antenna length is $L=684 \mathrm{~mm}$, the radius is $a=28 \mathrm{~mm}$, pitch angle is $\alpha=13.5^{\circ}$, and the wire radius is $r=0.3 \mathrm{~mm}$. The number of turns is, consequently, $N=16.2$. The total 
unfolded length of the wire is about $3 \mathrm{~m}$. For this particular antenna design, we have extensive experimental data that are in excellent agreement with the simulation results using program Awas [6]. This fact gave us confidence to base the analysis in this paper only on numerical models.

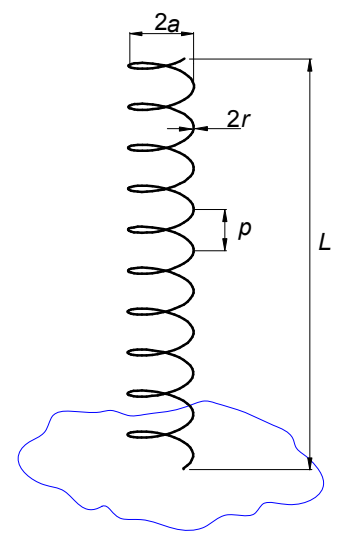

Figure 1: Helical antenna above infinite ground plane.

Figure 2 shows the computed antenna gain in the zenith direction, as a function of frequency. The gain has a broadband characteristic. In the frequency range $1.21-2.12 \mathrm{GHz}$, the gain is in the range $(12.2 \pm 1.5) \mathrm{dBi}$. Hence, according to [2], this particular antenna design corresponds to the WB3 design (wideband design with gain variations within $3 \mathrm{~dB}$ ). We want to point out that overlapping of the WB3 design data and the classical design recommendations is only accidental.

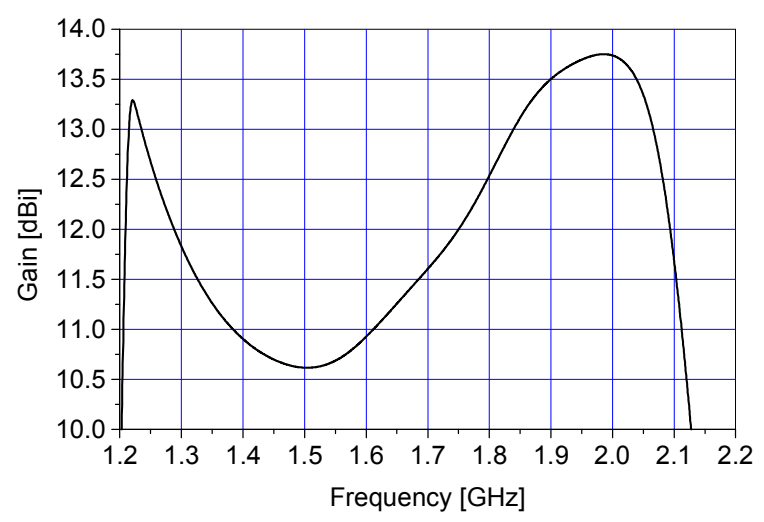

Figure 2: Gain versus frequency for antenna in Figure 1.

Figure 3 shows the radiation pattern of the antenna at several frequencies. The sidelobes are low only in the narrow band near $1.225 \mathrm{GHz}$. At higher frequencies, the sidelobes are highly pronounced and their level is as high as $6 \mathrm{~dB}$ below the main lobe.

The results clearly indicate that the antenna performance is not superb, although the antenna design follows the classical rules. The bandwidth of the gain is good, but the sidelobes are too high. For the same antenna length $(L)$ and narrower bandwidth, higher gain and better radiation pattern can be achieved. In [2], guidelines are given as how to select the optimal parameters for antennas above infinite ground plane in order to maximize the gain. The optimal pitch angle can be in a much wider range $\left(3^{\circ}-20^{\circ}\right)$ than according to the classical data. The optimal angle depends on both the wire radius and the antenna length.

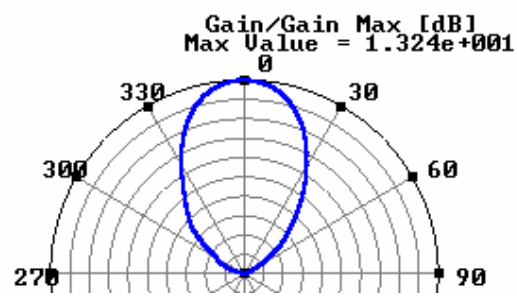

$1.225 \mathrm{GHz}$

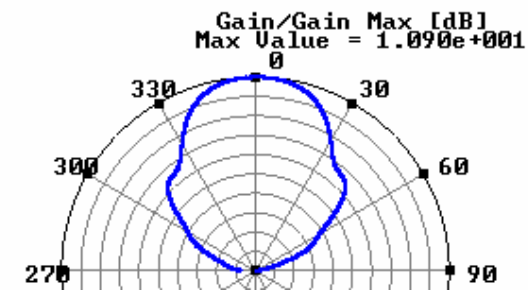

$1.4 \mathrm{GHz}$

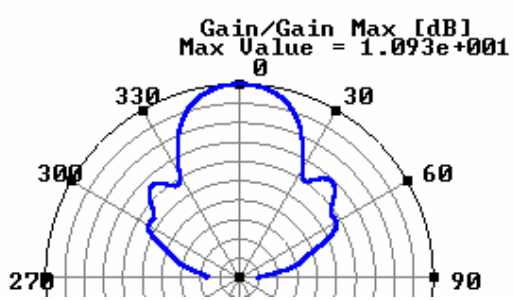

$1.6 \mathrm{GHz}$

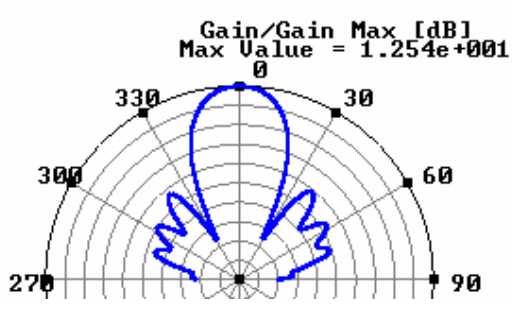

$1.8 \mathrm{GHz}$

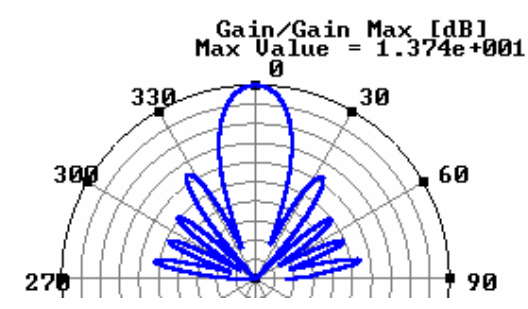

$2 \mathrm{GHz}$

Figure 3. Radiation pattern of antenna in Figure 1. Scale is $2 \mathrm{~dB}$ per division. 


\subsection{Finite-size ground conductor}

We showed in [3] that the antenna radiation pattern and the gain depend strongly on the shape and size of the ground conductor. Figure 4 depicts helical antennas with several shapes of the ground conductor. Figure 5 shows the gain of these antennas as a function of frequency. The helix is the same as selected in Subsection 2.1. The corresponding optimal size of the square plate is $b=1.5 \lambda$, where $\lambda=176 \mathrm{~mm}$. The optimal dimensions of the cup are $D=1 \lambda$ and $h=0.25 \lambda$. The optimal dimensions of the truncated cone are $D_{1}=0.75 \lambda, D_{2}=2.5 \lambda, h=0.5 \lambda$.

The conical reflector offers the highest gain. The increase of the gain with respect to the infinite ground plane exceeds $3 \mathrm{~dB}$.

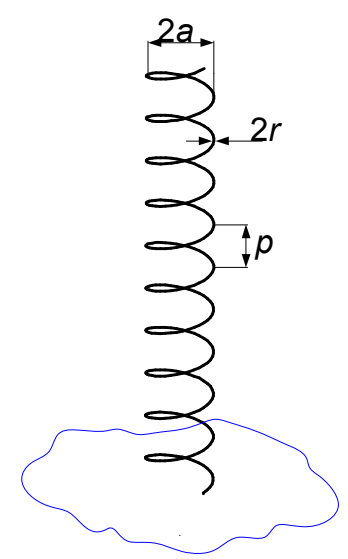

(a)

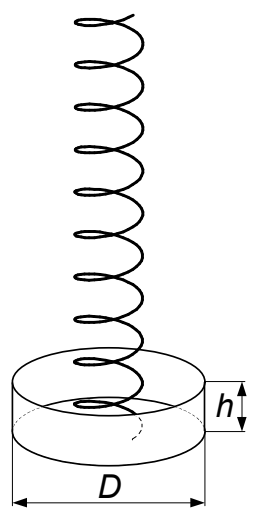

(c)

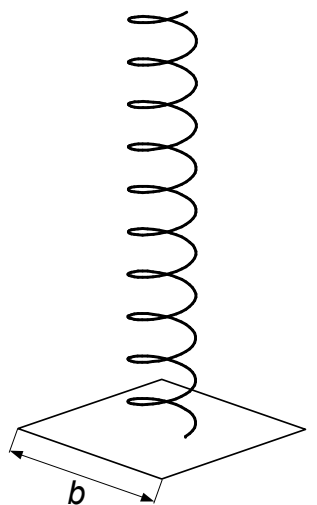

(b)

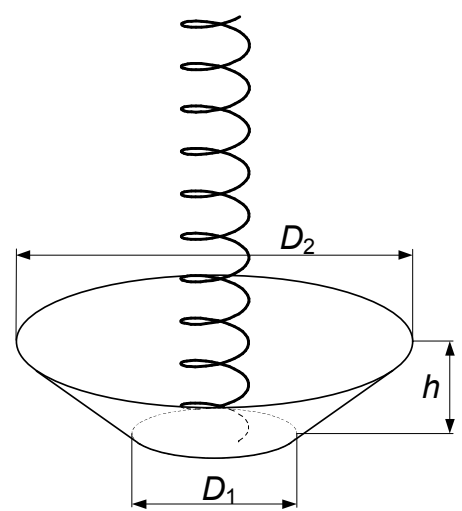

(d)
Figure 4. Helical antenna above (a) infinite ground plane, (b) square conductor, (c) cylindrical cup, and (d) truncated cone.

In [4], we optimized both the dimensions of the conical reflector and the dimensions of the helix to increase further the antenna gain. A wide range of cone heights was considered, up to $h=2 \lambda$. In some cases, the cone was as high as the helical antenna. The results show that the antennas above optimal conical ground conductor have even $8 \mathrm{~dB}$ higher gain than those above an infinite ground plane. Note that the optimal helical antenna with truncated cone from [3] (for $h=0.5 \lambda$ ) fits well into the data presented in [4].

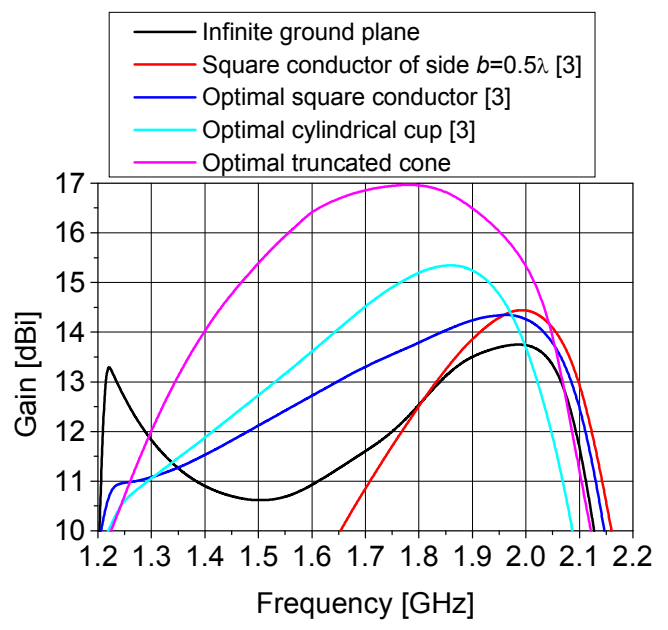

Figure 5. Gain for various shapes of the ground conductor.

Figure 6 compares various data for antenna gain. The first set is taken from [7]. It consists of compiled data for helical antennas and the gain estimation based on the HansenWoodyard condition. Figure 6 also presents data for optimal helical antennas above infinite ground plane taken from [2] for the narrowband (NB) and wideband (WB3) designs. Finally, data are given for optimal helical antennas with truncated cones [4] for two cone heights. Note that for tall cones, the length $(L)$ of the helix has practically no influence on the gain. This fact indicates that the cone is the main source of radiation, acting like a horn antenna [8].

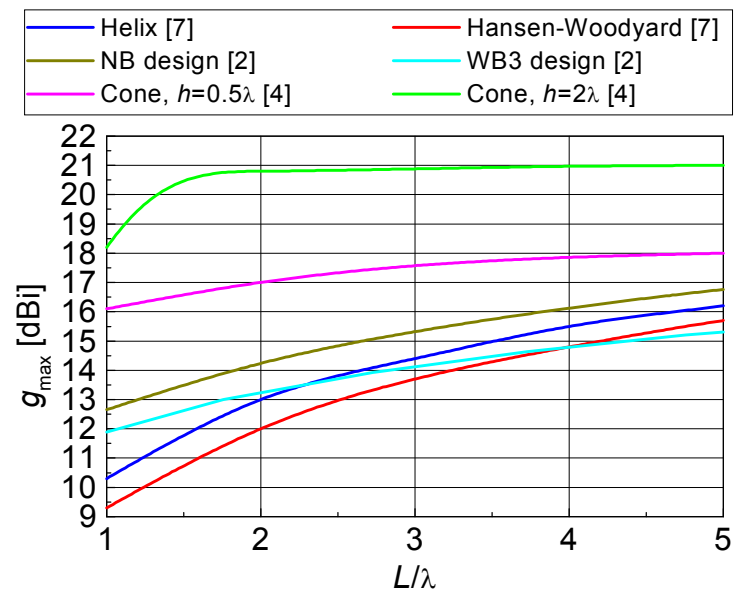

Figure 6. Gain comparison.

Although it has been known that proper design of the ground conductor (reflector) can increase the gain of the helical antenna, an open question remains about the physical cause for this increase. In the next sections, we try to answer this question. 


\section{Current distribution along helix}

According to the classical analysis of the helix as a guiding structure [9], four waves can be excited on it: two traveling waves with constant amplitudes, propagating in opposite directions, and two exponentially decaying waves, also propagating in opposite directions. To verify this theory, we analyze helical antennas numerically and extract the current distribution along the helically-wound wire.

Figure 7 shows the current distribution along the wire conductor of the helical antenna described in Subsection 2.1, located above an infinite ground plane. The operating frequency is $1.8 \mathrm{GHz}$.

The magnitude of the current is normalized with respect to the current at the feeding point. (The feeding point is localized on the periphery of the cylinder on which the wire is wound.) The phase slope is normalized so that a TEM wave propagating in a vacuum would have the normalized slope of 1 or -1 , depending on the direction of propagation.

A fast decaying current distribution is notable on the lowest two turns (feeding region). This resembles the classical exponentially-decaying wave. The current almost vanishes at the end of this region.

In the middle zone, the traveling-wave distribution dominates. The presence of waves traveling in opposite directions can be observed, though the standing-wave ratio is not large. One turn of the helix approximately contains two periods of the standing wave.

The current goes to zero at the tip of the antenna. Near the tip, a strong standing wave is visible.

Note that the antenna gain in the zenith direction is $12.5 \mathrm{dBi}$ (Figure 2).

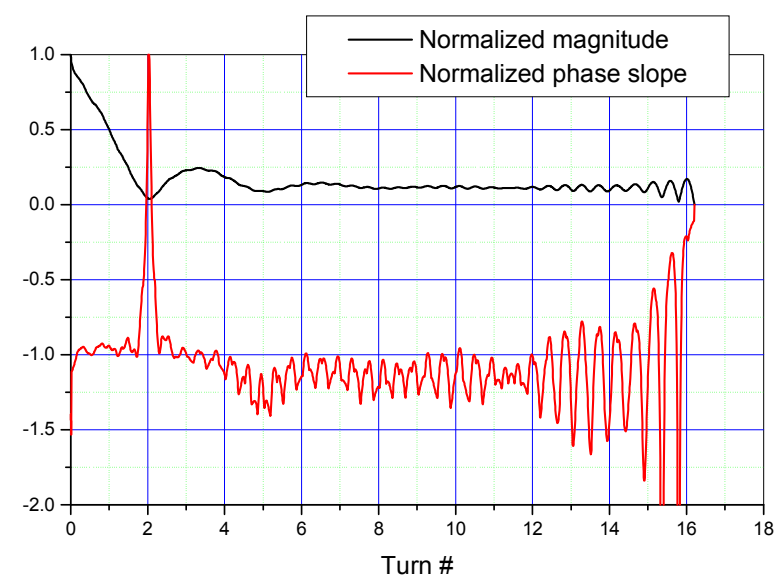

Figure 7. Current distribution of helical antenna above infinite ground (WB3 design).

Figure 8 shows the current distribution for the same antenna, but with the optimal truncated cone for $h=0.5 \lambda$ [3] instead of the infinite ground plane. The antenna gain is $17 \mathrm{dBi}$.
Compared to Figure 7, the magnitude of the traveling wave is now higher for about $50 \%$.

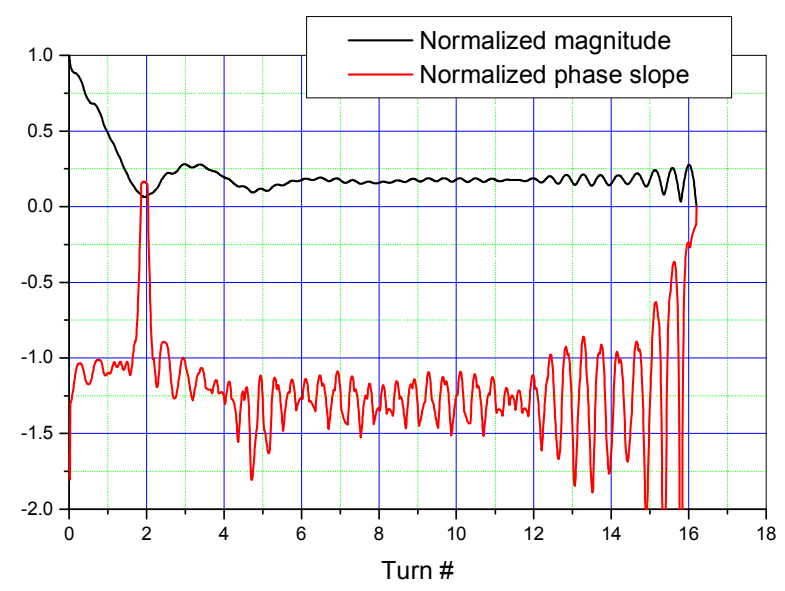

Figure 8. Current distribution of helical antenna with truncated cone.

In order to estimate the contribution of traveling wave on the radiation pattern of the helix antenna, in Figure 9 we compare the field produced by all turns of the helical antenna and the field evaluated by removing the contribution of the lowest 4 turns. The latter pattern practically takes into account only the contribution of the traveling wave. The antenna is located above an infinite ground plane and the relative radiation patterns are shown on a linear scale.

The traveling wave produces a relatively well-shaped radiation pattern. Hence, the strong sidelobes at low elevation angles must be created by the current of the lowest few turns, which confirms the conclusions in [10].

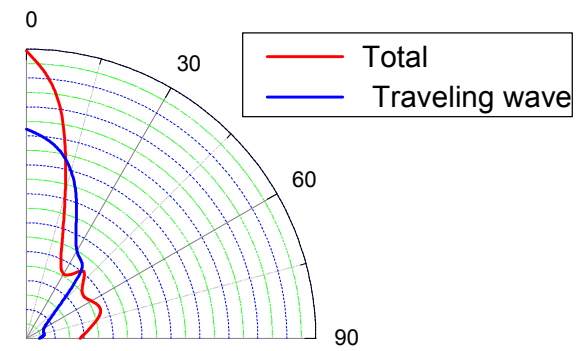

Figure 9. Typical radiation pattern of helical antenna and pattern produced by traveling wave.

Comparing Figures 7 and 8, the increase of the travelingwave magnitude may seem to be primarily responsible for the gain increase from $12.5 \mathrm{dBi}$ to $17 \mathrm{dBi}$. However, this reasoning disregards the fact that the input resistance of the antenna changes to a certain extent when the shape of the ground conductor is changed. In addition, the traveling wave yields lower sidelobes, but the main-beam width is larger. Our extensive computations for various helical antennas have not shown a correlation between the traveling-wave magnitude and the antenna gain. Examples were found where a weaker traveling wave corresponds to a higher-gain 
antenna, though enhancing the traveling wave did contribute to lowering the sidelobes. These findings motivate us to investigate other factors that affect the gain of helical antennas.

\section{Contributions to radiated field}

In order to determine other factors that affect the gain of a helical antenna, we evaluate the radiated fields created by various parts of the helical antenna and the ground conductor. We perform computations in the following way. The antenna, together with the ground conductor, is analyzed using program Awas [6]. An infinite ground plane is modeled in the program by images. All other ground conductors (cup, cone) are modeled by wire-grid structures, as shown in Figure 10. Once the current distribution in the wires is obtained, fields created by various portions of the system are evaluated using this current. In particular, contributions of the fields reflected from the infinite ground plane, fields created by the feeding region of the helix, fields created by the traveling-wave part of the helix, and fields created by the cup and cone are evaluated.

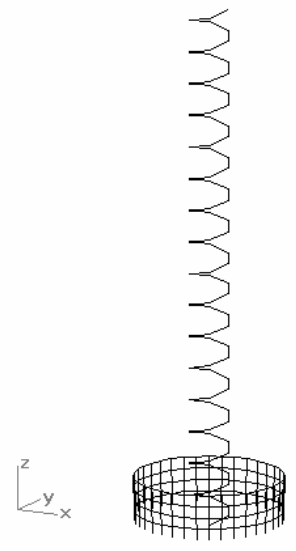

(a)

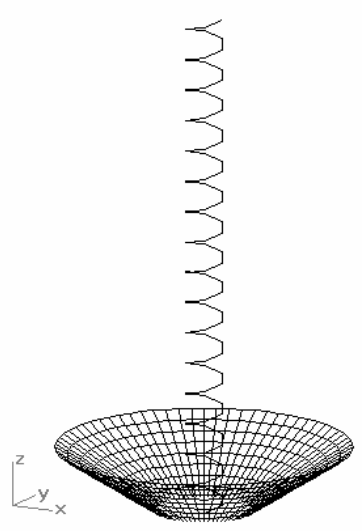

(b)
Figure 10. Helical antenna with wire-grid models of (a) cup and (b) cone.

\subsection{Infinite ground plane}

In this subsection, we consider helical antenna above an infinite ground plane. The fields produced by the currents and charges induced in the ground plane are evaluated by using the image of the helical wire (which is the original).

The performance of the helical antenna can also be visualized in terms of waves. We consider the helical wire as the primary source of the waves (fields). Any wave that travels downwards becomes reflected by the ground plane. Thereafter, it travels upwards, as if it comes from the image in the ground plane. The waves directly launched from the helix into the upper hemisphere interfere with the reflected waves, creating the resulting radiation pattern.
Figure 11 shows the relative radiation pattern (on a linear scale) produced by the helical wire (the original), the pattern created by the image in the ground plane, and the total field.
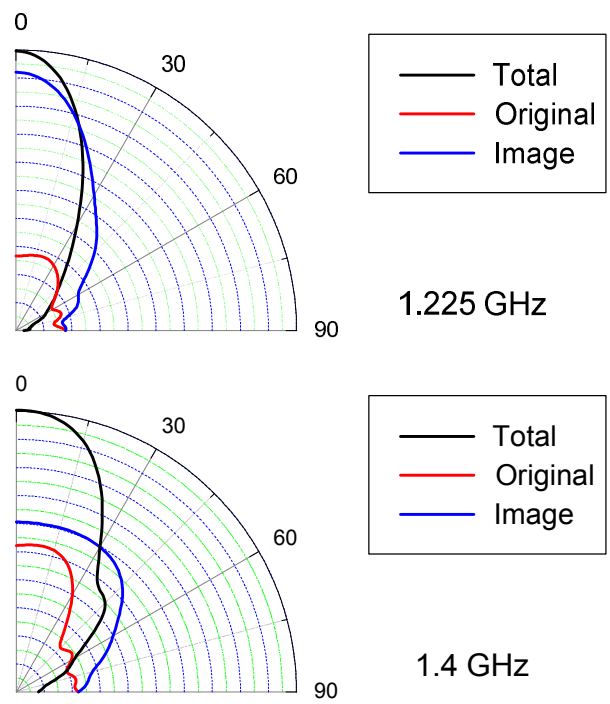

$1.4 \mathrm{GHz}$
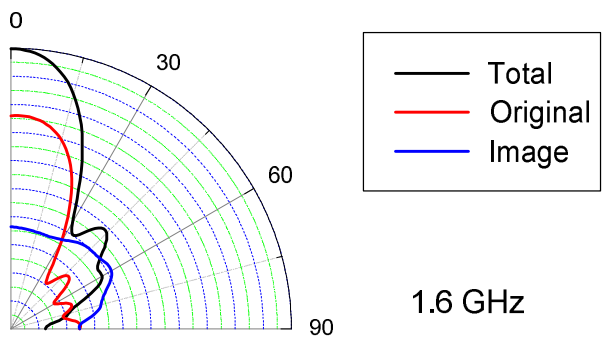

$1.6 \mathrm{GHz}$
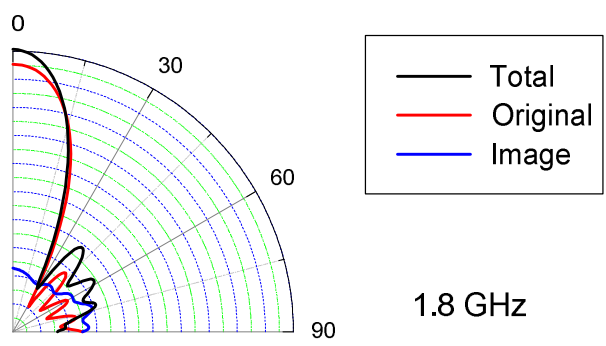

$1.8 \mathrm{GHz}$

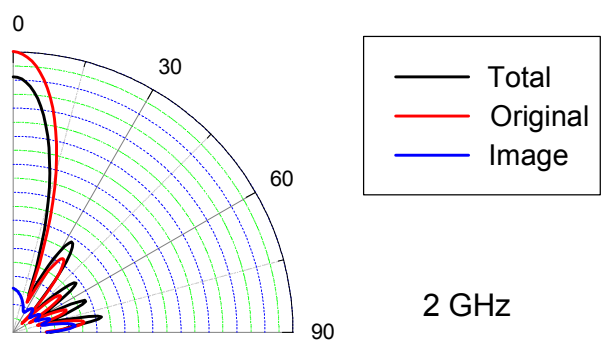

Figure 11. Contribution of infinite ground plane.

At higher frequencies (above about $1.7 \mathrm{GHz}$ ), the field produced by the ground plane is small. Other data confirm this conclusion. For example, in this frequency range, the antenna performs well even with a small flat ground plate (Figure 5). Additionally, the antenna gain in the upward direction does not significantly depend on the size of this plate [3]. At lower frequencies, however, the field reflected from the infinite ground plane is strong. Below about 
1.4 GHz, this field is even stronger than the field launched upwards from the helix. This fact explains the existence of the maximum of the antenna gain (Figures 2 and 5) at lower frequencies (around $1.225 \mathrm{GHz}$ ). Note that this maximum does not exist for antennas above small ground conductors (e.g., a small square plate), as can be seen in Figure 5.

\subsection{Feeding region and traveling-wave region}

The current distribution along the few lowest turns, next to the helix feed, does not resemble traveling waves (Figures 7 and 8). The field radiated by these turns is strong, but it is not directed upwards. Rather, it is almost omnidirectionally spread around, as shown in Figure 12 for $1.8 \mathrm{GHz}$. Similar conclusions apply to other frequencies.

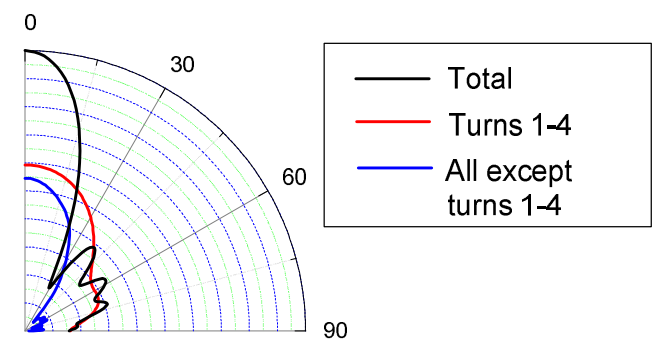

Figure 12. Contribution of the lowest four turns.

The fields in Figure 12 include the influence of the ground plane. At lower frequencies, the relative contribution of the lowest turns is larger than at higher frequencies. The lower turns generate a strong field that propagates downwards (towards the ground plane).

The currents and charges on the lowest few turns are the dominant source of the sidelobes at low elevation angles. These sources are located relatively close to the ground plane, i.e., they are within one wavelength for the classical values of the pitch angles (about $13^{\circ}$ ). Hence, their field can be intercepted relatively easily by a surrounding metallic structure, like the rim of the cup or the cone (Figures $4 \mathrm{c}, \mathrm{d}$ and 10 ), and diffracted or reflected in a more favorable direction. This is a possible explanation for the action of the cup and cone on improving the radiation pattern of the helical antenna.

If the helix pitch is decreased near the feed, the field produced by the lowest few turns is reduced. This reduction can be attributed to partial field cancellation by the images in the ground plane, as well as to the reduced current momentum because of the lower elevation of these turns. This explains the favorable effect of taking nonuniform (denser) turns in the excitation region, as well as the effect of various travelingwave launchers used to feed helical antennas (e.g., [10]).

This effect can also explain the good performance of helical antennas with a small pitch [2]. Such antennas yield a good narrowband (NB) performance, exhibiting high gain. As an example, let us consider a helical antenna that has the same length as the antenna introduced in Subsection 2.1 ( $L=684 \mathrm{~mm}$ ). However, the narrowband antenna has a notably smaller pitch angle $\left(\alpha=5.2^{\circ}\right)$, corresponding to
$N=42$ turns. The helix radius is $a=24 \mathrm{~mm}$, and the wire radius is $r=0.3 \mathrm{~mm}$. The total unfolded length of the wire is about $6.7 \mathrm{~m}$. Figure 13 shows the current distribution along this antenna at $1.8 \mathrm{GHz}$, where the antenna gain is as high as $16.4 \mathrm{dBi}$.

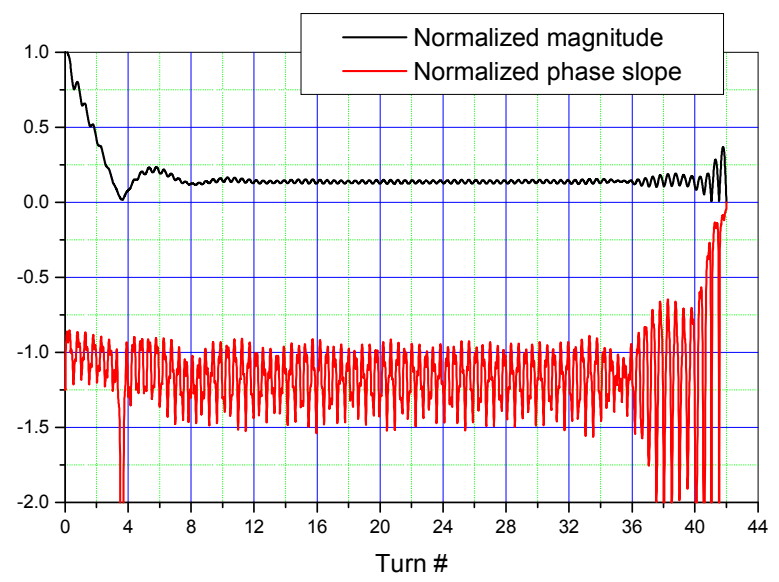

Figure 13. Current distribution of helical antenna above infinite ground (NB design).

The height of the feeding region for the NB design antenna is smaller than the height for the WB3 design antenna (Figure 7). In particular, the dip in the current distribution for the NB design antenna (Figure 13) is located at $59 \mathrm{~mm}$ above the ground plane, whereas the dip for the WB3 design antenna (Figure 7) is at $87.5 \mathrm{~mm}$. Consequently, for the NB design antenna, the traveling-wave region starts at a lower elevation. The radiation pattern has lower sidelobes (Figure 14) because the feeding region creates weaker radiation for low elevation angles. This can also be seen in Figure 15 on a linear scale. (Note that Figures 14 and 15 correspond to the frequency of $1.7 \mathrm{GHz}$.)

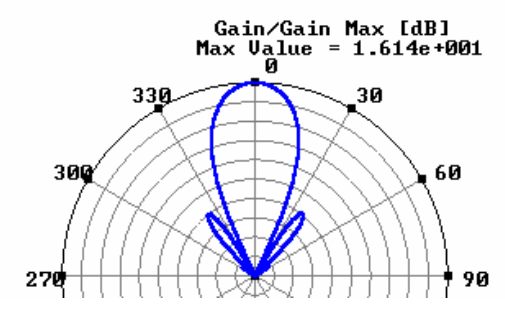

Figure 14. Radiation pattern of narrowband antenna. Scale is $2 \mathrm{~dB}$ per division.

Figure 16 shows the gain of the narrowband helical antenna, as a function of frequency. The gain is much higher than the gain of the antenna introduced in Subsection 2.1, and it approaches the performance of the antenna with conical reflector. The price to pay is, however, substantially narrower bandwidth. 


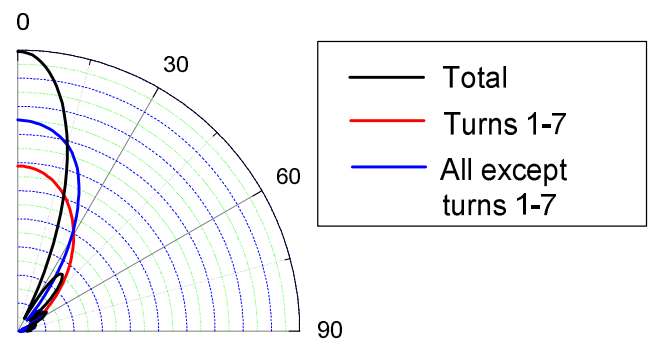

Figure 15. Contribution of the lowest seven turns for narrowband antenna.

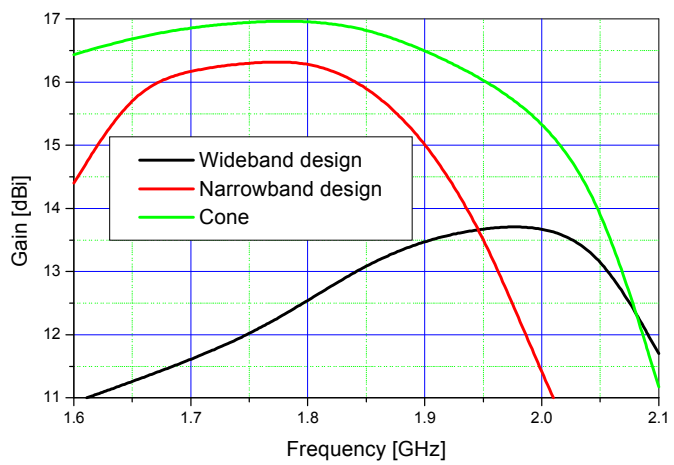

Figure 16. Gain of helical antennas for WB3 and NB design [2], and of helical antenna with optimal conical ground $(h=0.25 \lambda)[3]$.

The performance of the narrowband antenna is almost unaffected by the shape of the ground conductor. A cup or a cone cannot significantly change the antenna gain because the antenna radiation is relatively weak for low elevation angles, so the ground does not intercept significant energy.

\subsection{Cup and cone}

The cup and cone shown in Figure 4c,d substantially improve the radiation pattern and gain of the helical antenna introduced in Subsection 2.1, as illustrated in Figure 17. This Figure shows the radiation pattern of the helical antenna with the optimal cone from [3]. Compared to the patterns in Figure 3, the antenna has narrower main lobe and substantially lower sidelobes.

One effect is common for the cup and the cone: they intercept the radiation coming from the lowest turns of the helix antenna and do not allow it to progress horizontally. Hence, they clear the sidelobes at low elevation angles. This is illustrated in Figure 18, where fields on a linear scale (at $1.8 \mathrm{GHz}$ ) are plotted. The four lowest turns of the helix produce strong fields near the ground plane. However, these fields are cancelled out by the fields created by cup, viz. the cone, as shown in Figure 19.

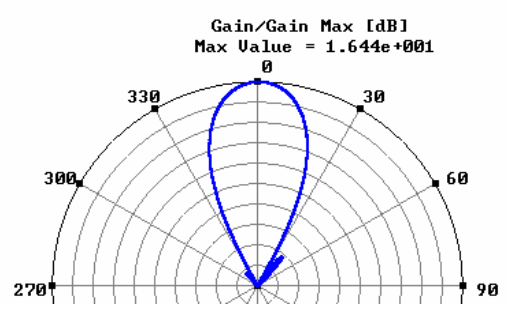

$1.6 \mathrm{GHz}$

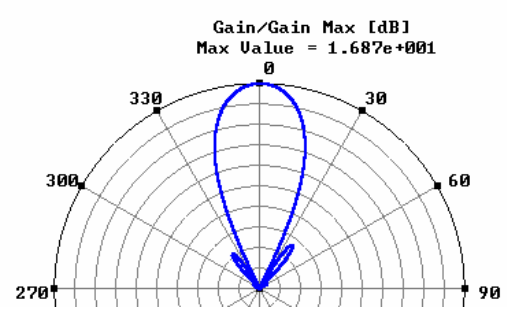

$1.7 \mathrm{GHz}$

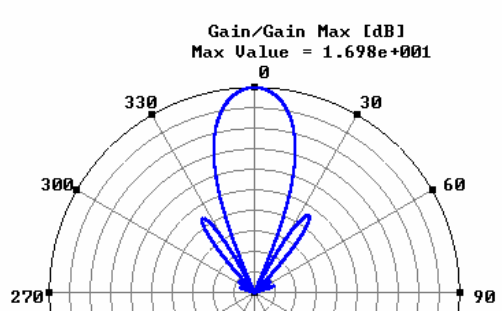

$1.8 \mathrm{GHz}$

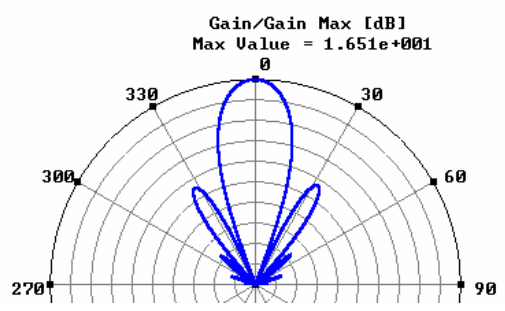

$1.9 \mathrm{GHz}$

Figure 17. Radiation pattern of helical antenna with conical ground conductor $(h=0.5 \lambda)$. Scale is $2 \mathrm{~dB}$ per division.

The cone, however, has another effect, not seen with the cup. When the size of the cone is large, the cone resembles a horn antenna. The helical antenna is placed within the horn and it feeds the horn. The resulting gain is much higher than the gain obtained by collecting spillover fields generated by the helix. The results presented in [4] show that this gain is close to the gain obtained by a classical horn antenna.

\section{Conclusions}

We investigated uniformly-wound helical antennas above various grounds, with aim to determine various factors that improve the antenna radiation pattern and gain. We analyzed all effects using a program for antenna computer simulation 
[6]. We evaluated the current distribution in the helix antenna and the ground conductor, and calculated contributions of various parts of the system.

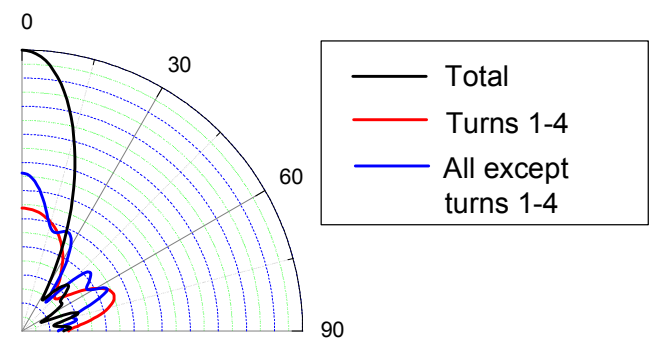

Cup

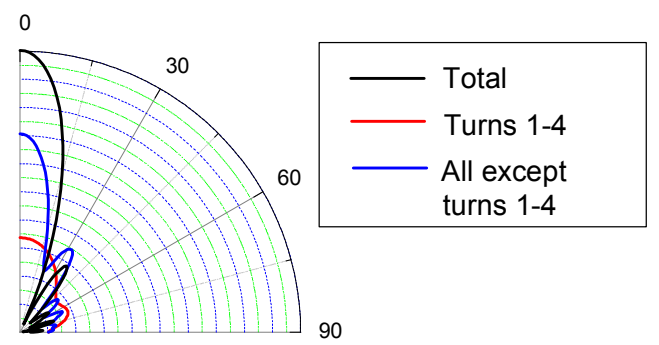

Cone

Figure 18. Contribution of the lowest four turns.

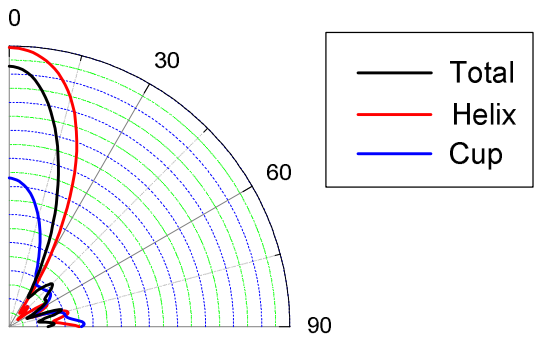

Cup

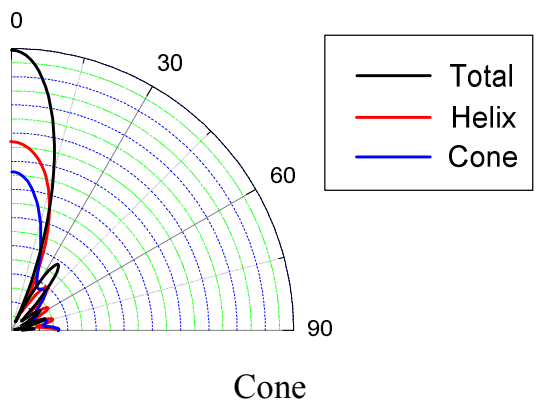

Figure 19. Contributions of helix and ground conductor.

We established that an infinite ground plane has favorable effect at lower frequencies. It can make the performance of some helical antennas more broadband by reflecting waves launched from the helix downwards. However, broadband helical antennas have lower peak gain than narrowband antennas.

The major source of spillover is the current in the lowest few turns of the helix. This current creates strong sidelobes at low elevation angles. Increasing the intensity of the traveling wave on the helical antenna yields radiation patterns with lower sidelobes.

A cylindrical ground conductor with a rim (a cup) and a conical ground conductor prevent propagation of the spillover fields in horizontal directions and direct them upwards. A large cone has an additional favorable effect, as it acts like a horn antenna and further increases the gain in the zenith direction.

The radiation pattern is significantly improved if the radiation from the lowest few turns is suppressed. This can be achieved by using various launchers presented in the literature or simply by reducing the helix pitch. For such antennas, the influence of the ground conductor on the radiation pattern is small.

\section{Acknowledgement}

This work was supported in part by the Serbian Ministry of Science and by the COST Action IC0603.

\section{References}

[1] J. D. Kraus, "Helical beam antennas", Electronics, 20, pp. 109-111 (1947).

[2] A. R. Djordjevic, A. G. Zajic, M. M. Ilic, and G. L. Stuber, "Optimization of helical antennas", IEEE Antennas and Propagation Magazine, 48, pp. 107-115 (2006).

[3] A. R. Djordjevic, A. G. Zajic, and M. M. Ilic, "Enhancing the gain of helical antennas by shaping the ground conductor", IEEE Antennas and Wireless Propagation Letters, 5, pp. 138-140 (2006).

[4] D. I. Olcan, A. G. Zajic, M. M. Ilic, and A. R. Djordjevic, "On the optimal dimensions of helical antenna with truncated-cone reflector", Proceedings of $E u C A P$, ESA SP-626 (2006).

[5] J. D. Kraus, Antennas, New York: McGraw-Hill, 1988.

[6] A. R. Djordjević, M. B. Baždar, T. K. Sarkar, and R. F. Harrington, AWAS for Windows: Analysis of Wire Antennas and Scatterers, Software and User's Manual, Boston: Artech House (2002).

[7] T. A. Milligan, Modern Antenna Design, Hoboken, N.J.: J. Wiley, 2005, Figure 10-2.

[8] K. R. Carver, "The helicone - a circularly polarized antenna with low sidelobe level", Proceedings of IEEE, 55, p. 559 (1967).

[9] R. E. Collin, Foundations for Microwave Engineering, New York: McGraw-Hill, 1992.

[10]D. J. Angelakos and D. Kajfez, "Modifications on the axial-mode helical antenna", Proceedings of IEEE, 55, pp. 558-559 (1967). 